\title{
RINGS AND GROUPS WITH COMMUTING POWERS
}

\author{
HAZAR ABU-KHUZAM \\ ADIL YAQUB \\ DEPARTMENT OF MATHEMATICS \\ PETROLEUM UNIVERSITY \\ SAUDI ARABIA
}

(Received March 5, 1979)

ABSTRACT. Let $\mathrm{n}$ be a fixed positive integer. Let $\mathrm{R}$ be a ring with identity which satisfies (i) $x^{n} y^{n}=y^{n} x^{n}$ for all $x, y$ in $R$, and (ii) for $x, y$ in $R$, there exists a positive integer $k=k(x, y)$ depending on $x$ and $y$ such that $x^{k} y^{k}=y^{k} x^{k}$ and $(n, k)=1$. Then $R$ is commutative. This result also holds for a group $G$. It is further shown that $R$ and $G$ need not be commutative if any of the above conditions is dropped.

KEY WORDS AND PHRASES. ring, group, center, Jacobson radical, commutative. 1980 MATHEMATICS SUBJECT CLASSIFICATION CODES: Primary 16A70, 20F10; Secondary $16 \mathrm{~A} 38$.

1. INTRODUCTION.

Throughout this section, $\mathrm{n}$ is a fixed positive integer. The main theorem of this section is the following:

THEOREM 1. Let $G$ be a group such that (i) $x^{n} y^{n}=y^{n} x^{n}$ for $\underline{\text { all }} x, y \underline{\text { in }}$

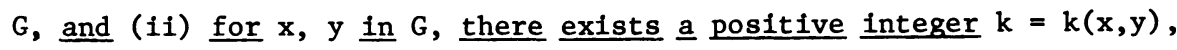


depending on $x$ and $y$ such that $x^{k} y^{k}=y^{k} x^{k}$ and $(n, k)=1$. Then $G$ is commutative.

In preparation for the proof of Theorem 1, we first prove the following Lemma LEMA 1. Let $G$ be as in Theorem 1. Then for every finite subset $F$ of $G$, there exists a positive integer $N=N(F)$, depending on $F$ such that $x^{N} y^{N}=y^{N} x^{N}$, and $x^{N+1} y^{N+1}=y^{N+1} x^{N+1}$ for al1 $x, y$ in $F$.

PROOF. Let $x_{1}, y_{1}, x_{2}$, and $y_{2} \varepsilon G$. Then by condition (ii) above there exist positive integers $k_{1}=k_{1}\left(x_{1}, y_{1}\right), k_{2}=k_{2}\left(x_{2}, y_{2}\right)$, both relatively prime to $n$, such that

$$
x_{1}^{k_{1}} y_{1}^{k_{1}}=y_{1}^{k_{1} x_{1}} \text { and } x_{2}^{k_{2} y_{2}}=y_{2}^{k_{2} x_{2}} \text {. }
$$

Let $k=k_{1} k_{2}$. Then, clearly $k$ is relatively prime to $n$, and

$$
x_{1}^{k} y_{1}^{k}=y_{1}^{k} x_{1}^{k}, \quad x_{2}^{k} y_{2}^{k}=y_{2}^{k} x_{2}^{k} .
$$

It follows easily, that for any finite subset $F$ of $G$ there exists a positive integer $k=k(F)$ which is relatively prime to $n$ and, $x^{k} y^{k}=y^{k} x^{k}$ for all $x$, $y$ in F. From (i) we know that $x^{n} y^{n}=y^{n} x^{n}$ for all $x, y$ in $G$. Since $k$ and $n$ are relatively prime, $r k-s n=1$ for some positive integers $r$ and s. If $N=$ sn then $N+1=r k$ and 1 follows that $x^{N} y^{N}=y^{N} x^{N}, x^{N+1} y^{N+1}=$ $y^{N+1} x^{N+1}$, for all $x, y$ in $F$. Clearly, $N$ depends on $F$. This proves Lemma 1.

PROOF OF THEOREM 1. Let $x, y \varepsilon G$, and let $F=\{x, y, x y, y x\}$. Then by Lemma 1 there exists a positive integer $N=N(F)$ such that $a^{N} b^{N}=b^{N} a^{N}$ and $a^{N+1} b^{N+1}=b^{N+1} a^{N+1}$ for $a l 1 a, b$ in F. Also, $x, y \varepsilon F$ implies $x^{N} y^{N}=y^{N} x^{N}$ and $x^{N+1} y^{N+1}=y^{N+1} x^{N+1}$. Hence $x^{N}=y^{-N} x^{N} y^{N}$ and $x^{N+1}=y^{-(N+1)} x^{N+1} y^{N+1}$. Now $x=x^{N+1} \cdot x^{-N}=y^{-(N+1)} x^{N+1} y^{N+1} y^{-N} x^{-N} y^{N}$, and hence

$$
x=y^{-(N+1)} x^{N+1} y x^{-N_{y} N} \text {. }
$$

Multiplying equation (1) by $y$ from the right we get

$$
x y=y^{-(N+1)} x^{N+1} y x^{-N} y^{N+1}
$$

and thus 


$$
(x y)=y^{-(N+1)} x^{N+1}(y x) x^{-(N+1)} y^{N+1}
$$

Raising both of the above equation to the power $(\mathrm{N}+1)$ we get

$$
(\mathrm{xy})^{\mathrm{N}+1}=\mathrm{y}^{-(\mathrm{N}+1)} \mathrm{x}^{(\mathrm{N}+1)}(\mathrm{yx})^{\mathrm{N}+1} \mathrm{x}^{-(\mathrm{N}+1)} \mathrm{y}^{\mathrm{N}+1} \text {. }
$$

Hence $(x y)^{N+1}=(y x)^{N+1}$, since $x, y$ and $y x \in F$. Multipling equation (1) by y from the left we get

$$
y x=y^{-N} x^{N+1} y^{-N} y^{N}
$$

and hence

$$
y x=y^{-N} x^{N}(x y) x^{-N} y^{N} .
$$

Raising both sides of the above equation to the power $\mathrm{N}$ we get

$$
(y x)^{N}=y^{-N} x^{N}(x y)^{N} x^{-N} y^{N} .
$$

But since $x, y, x y \varepsilon F$ we get

$$
(y x)^{N}=(x y)^{N} \text {. }
$$

Now $(x y)=(x y)^{N+1} \cdot(x y)^{-N}=(y x)^{N+1} \cdot(y x)^{-N}=y x$, and hence $G$ is abelian

2. RINGS.

Throughout this section, $\mathrm{R}$ is an associative ring with identity 1 and $[x, y]=x y-y x$ for $x, y$ in $R$.

In preparation for the proof of the main theorem of this section, we first prove the following:

LEMMA 2. Suppose $x$ and $y \underline{\text { are elements }}$ of $R$, satisfying $k x^{m}[x, y]=0$ and $k(x+1)^{m}[x, y]=0$, for some positive integers $k$ and $m$. Then $k[x, y]=0$.

PROOF. Expanding $(x+1)^{\mathrm{m}}$ in $k(x+1)^{\mathrm{m}}[\mathrm{x}, \mathrm{y}]=0$ we get

$$
k x^{m}[x, y]+k c_{m-1} x^{m-1}[x, y]+\ldots+k c_{1} x[x, y]+k[x, y]=0 .
$$

By hypothesis, $\mathrm{kx}^{\mathrm{m}}[\mathrm{x}, \mathrm{y}]=0$, so if $\mathrm{m}=1$ then the result follows immediately from (2). Suppose $m>1$. Now, multiply equation (2) by $x^{m-1}$ from the left, and use the hypothesis $\mathrm{kx}^{\mathrm{m}}[\mathrm{x}, \mathrm{y}]=0$ to get $\mathrm{kx}^{\mathrm{m}-1}[\mathrm{x}, \mathrm{y}]=0$. Equation (2) becomes:

$$
k c_{m-2} x^{m-2}[x, y]+\ldots+k c_{1} x[x, y]+k[x, y]=0 .
$$


If $\mathrm{m}=2$, then since $\mathrm{kx}^{\mathrm{m}-1}[\mathrm{x}, \mathrm{y}]=0$, the result follows immediately from (2) Suppose $m>2$. Now, multiplying equation (3) by $x^{m-2}$ from the left, and use the fact that $\mathrm{kx}^{\mathrm{m}-1}[\mathrm{x}, \mathrm{y}]=0$ to get $\mathrm{kx}^{\mathrm{m}-2}[\mathrm{x}, \mathrm{y}]=0$. Continue this process until we get $k[x, y]=0$, which proves the lemma.

Now we will prove the analogue to Theorem 1 for rings.

THEOREM 2. Let $\underline{\mathrm{n}}$ be a fixed positive integer. Suppose that $R \underline{\text { is }}$ an associative ring with identity 1 such that (1) $x^{n} y^{n}=y^{n} x^{n}$ for $\underline{\text { all }} x, y$ in

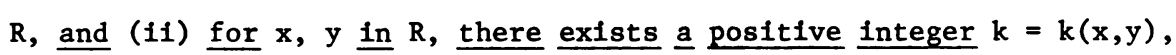
depending on $x$ and $y$ which is relatively prime to $n$ such that $x^{k} y^{k}=y^{k} x^{k}$. Then $R$ is commutative.

In preparation for the proof of Theorem 2, we first state the following 1emma.

LEMMA 3. Let $R$ be as in Theorem 2. Then for every finite subset $F$ of $R$, there exists a positive integer $N=N(F)$, depending on $F$ such that $x^{N} y^{N}=y^{N} x^{N}$ and $x^{N+1} y^{N+1}=y^{N+1} x^{N+1}$ for $\underline{\text { all }} x, y$ in $F$.

PROOF. Exactly the same as the proof of Lemma 1.

PROOF OF THEOREM 2. Throughout the proof, $J, Z$ and $R^{*}$ will denote respectively the Jacobson radical, the center, and the group of units of $R$. The proof is broken into the following partial results.

CLAIM 1. $R^{*}$ is abelian, and $R / J$ is commutative.

PROOF. $R^{*}$ is abelian, by Theorem 1 . It is easy to see that the above hypotheses are inherited by subrings and by homomorphic images of $R$. Also, the above hypotheses imply that the idempotents are in the center, and hence no $m \times m$ complete matrix ring over a division ring can satisfy our hypotheses for $m>1$. So by the Jacobson Density Theorem, a primitive ring satisfying our hypotheses must be a division ring, and hence is commutative, by Theorem 1 . Also, R/J is semisimple, and hence it is a subdirect sum of primitive rings. 
So $\mathrm{R} / \mathrm{J}$ is commutative.

CLAIM 2. $\mathrm{J}$ is commutative, and $\mathrm{J}^{2} \subseteq \mathrm{z}$.

PROOF. Suppose $a, b \in J$. Then $a+1$ belong to $R^{*}$, and hence they commute by Claim 1 . So $a b=b a$, and $J$ is commutative. For $x \in R$, we have

$$
(a b) x=a(b x)=(b x) a=b(x a)=(x a) b=x(a b) .
$$

So $a b \in z$, and hence $J^{2} \subseteq z$.

For the remainder of this proof, let a $\varepsilon J, y \varepsilon R$. Let $F^{\prime}=\{a, y, a+1, y+a$, $y+1\}$. Then by Lemma 3 , there exists a positive integer $N=N\left(F^{\prime}\right)$ such that $c^{N} d^{N}=d^{N} c^{N}$ and $c^{N+1} d^{N+1}=d^{N+1} c^{N+1}$ for all $c, d \varepsilon F^{\prime}$.

CLAIM 3. $N\left[a, y^{N}\right]=0=(N+1)\left[a, y^{N+1}\right]$.

PROOF. Since a $\varepsilon J, u=1+a \varepsilon R^{*}$. Also, $R / J$ is commutative, and hence $\left[\mathrm{u}, \mathrm{y}^{\mathrm{N}}\right] \in \mathrm{J}$. So $\mathrm{u}$ commutes with $\left[\mathrm{u}, \mathrm{y}^{\mathrm{N}}\right]$, and hence by induction, $0=\left[\mathrm{u}^{\mathrm{N}}, \mathrm{y}^{\mathrm{N}}\right]=$ $=\mathrm{Nu}^{\mathrm{N}-1}\left[\mathrm{u}, \mathrm{y}^{\mathrm{N}}\right]$. This implies that $\mathrm{N}\left[\mathrm{u}, \mathrm{y}^{\mathrm{N}}\right]=0$, since $\mathrm{u}$ is invertible. Hence $N\left[a, y^{N}\right]=0$. Similarly, since $\left[u^{N+1}, y^{N+1}\right]=0$, we have $(N+1)\left[a, y^{N+1}\right]=0$.

CLAIM 4. $N[a, y]=0$ and $\left[a, y^{N+1}\right]=0$.

PROOF. Since $y \in F^{\prime}$ and $y+a \varepsilon F^{\prime}$, we have

$$
\left[(y+a)^{N+1}, y^{N+1}\right]=0 \text {. }
$$

Since $a \in J, a^{2} \varepsilon z$, and hence the only terms in the expansion of $(y+a)^{N+1}$ which do not commute with $\mathrm{y}^{\mathrm{N}+1}$ are those involving one a. So

$$
0=\left[(y+a)^{N+1}, y^{N+1}\right]=\left[y^{N} a+y^{N-1} a y+\ldots+y a y^{N-1}+a y^{N}, y^{N+1}\right] \text {. }
$$

By Claim 3, $\mathrm{Ny}^{\mathrm{N}} \mathrm{a}=\mathrm{Nay}^{\mathrm{N}}$, and so we can write the following:

$$
\begin{gathered}
N\left(y^{N} a+y^{N-1} a y+\ldots+y_{a y}{ }^{N-1}+a y^{N}\right) y^{N+1} \\
=N\left(y^{2 N} a y+y^{2 N-1} a{ }^{2}+\ldots+y^{N+1} a y^{N}\right)+N a y^{2 N+1},
\end{gathered}
$$

and 


$$
\begin{aligned}
& \mathrm{Ny}^{\mathrm{N}+1}\left(\mathrm{y}^{\mathrm{N}} \mathrm{a}+\mathrm{y}^{\mathrm{N}-1} \mathrm{ay}+\ldots+\mathrm{yay}^{\mathrm{N}-1}+a y^{\mathrm{N}}\right) \\
& =\mathrm{Ny} \\
& 2 \mathrm{~N}+1 \mathrm{a}+\mathrm{N}\left(\mathrm{y}^{2 \mathrm{~N}} \mathrm{ay}+\ldots+\mathrm{y}^{\mathrm{N}+1} \mathrm{ay}^{\mathrm{N}}\right) .
\end{aligned}
$$

By (4), the expressions on the left of the above two equations are equal, and hence we have $\mathrm{Nay}^{2 \mathrm{~N}+1}=\mathrm{Ny}^{2 \mathrm{~N}+1} \mathrm{a}$. But $\mathrm{Ny}^{2 \mathrm{~N}} \mathrm{a}=\mathrm{Nay}^{2 \mathrm{~N}}$, by Claim 3. So $\mathrm{Ny}^{2 \mathrm{~N}}[\mathrm{y}, \mathrm{a}]$ $=0$. Now, since $y+1 \varepsilon F$, the same result holds for $(y+1)$ instead of $y$. So $N(y+1)^{2 N}[y, a]=0$, and hence by Lemma 2 , we have $N[y, a]=0$. By Claim 3 , $(N+1)\left[a, y^{N+1}\right]=0$, and hence $N\left[a, y^{N+1}\right]+\left[a, y^{N+1}\right]=0$. But $N\left[a, y^{N+1}\right]=0$, since $N\left[a, y^{N}\right]=0$ and $N[a, y]=0$. Therefore, $\left[a, y^{N+1}\right]=0$.

Now we can complete the proof of Theorem 2. Since $y+a$ and $y$ belong to $F^{\prime},\left[(y+a)^{N}, y^{N}\right]=0$. Also, $a^{2} \varepsilon z$. Hence,

$$
\left[y^{N-1} a+y^{N-2} a y+\ldots+y^{N} y^{N-2}+a y^{N-1}, y^{N}\right]=0 \text {. }
$$

By Claim 4, ay ${ }^{N+1}=y^{N+1} a$, and hence we can write the following:

$$
\begin{aligned}
& \left(y^{N-1} a+y^{N-2} a y+\ldots+y a y^{N-2}+a y^{N-1}\right) y^{N} \\
& =y^{N-1} a y^{N}+\left(y^{2 N-1} a+y^{2 N-2} a y+\ldots+y^{N+1} a y^{N-2}\right),
\end{aligned}
$$

and

$$
\begin{gathered}
y^{N}\left(y^{N-1} a+y^{N-2} a y+\ldots+y^{N} y^{N-2}+a y^{N-1}\right) \\
=\left(y^{2 N-1} a+y^{2 N-2} a y+\ldots+y^{N+1} a y{ }^{N-2}\right)+y^{N} a y^{N-1} .
\end{gathered}
$$

By (5), the above two expressions on the left are equal, and hence $y^{N-1}$ ay ${ }^{N}=$ $y^{N}{ }_{a y}{ }^{N-1}$. Multiply this equation from the left and right by $y$ to get $y^{N}$ ay ${ }^{N+1}=$ $y^{N+1} a y^{N}$. Then, using Claim 4, we get $y^{2 N+1} a=a y^{2 N+1}$. But from Claim 4, we have $a y^{2 N+2}=y^{2 N+2} a$. So $y^{2 N+1}[a, y]=0$. Now since $y+1 \varepsilon F^{\prime}$, the above result holds for $y+1$, and hence $(y+1)^{2 N+1}[a, y]=0$. 
But then by Lemma 2, we have $[\mathrm{a}, \mathrm{y}]=0$. Th1s proves that $\mathrm{J} \subseteq \mathrm{z}$. Now, let $\mathrm{x}$, $y \varepsilon R$. Then $[x, y] \varepsilon J$, since $R / J$ is commutative. Therefore, $[x, y] \varepsilon z$, since $\mathrm{J} \subseteq \mathrm{z}$. Let $\mathrm{F}^{-}=\{\mathrm{x}, \mathrm{y}, \mathrm{x}+1, \mathrm{y}+1\}$. Then by Lemma 3 , there exists a positive integer $k=k\left(F^{\prime \prime}\right)$ such that $c^{k} d^{k}=d^{k} c^{k}$ and $c^{k+1} d^{k+1}=d^{k+1} c^{k+1}$ for all $c$, $\mathrm{d} \varepsilon \mathrm{F}^{\prime}$. Recall that $[\mathrm{x}, \mathrm{y}] \varepsilon \mathrm{z}$ and hence, by induction, we see that $0=\left[\mathrm{x}^{\mathrm{k}}, \mathrm{y}^{\mathrm{k}}\right]=$ $k x^{k-1}\left[x, y^{k}\right]$. Since $x+1 \varepsilon F^{\prime}$, the same result holds for $x+1$ replacing $x$, and hence $k(x+1)^{k-1}\left[x, y^{k}\right]=0$. Then, by Lemma 2 we have $k\left[x, y^{k}\right]=0$. So, by induction as in above, $0=k\left[x, y^{k}\right]=k^{2} y^{k-1}[x, y]$. Again, since $y+1 \varepsilon F^{\prime}$, the same result holds for $y+1$ replacing $y$, so $k^{2}(y+1)^{k-1}[x, y]=0$. Applying Lemma 2, we get $k^{2}[x, y]=0$. Similarly, since $\left[x^{k+1}, y^{k+1}\right]=0$, we get $(k+1)^{2}$ $[x, y]=a$, and hence $[x, y]=0$ which proves Theorem 2 .

We conclude with the following remarks:

REMARK 1. A finite nonabelian group shows that we cannot drop any of the hypotheses in Theorem 1 .

REMARK 2. Theorem 2 need not be true if $R$ has no identity. For, let $R$ be a finite nil non-commutative ring.

REMARK 3. Theorem 2 need not be true if either condition (i) or (ii) is deleted. For, let

$$
R=\left(\begin{array}{lll}
a & b & c \\
0 & a & d \\
0 & 0 & a
\end{array}\right): a, b, c, d \in G F(2) \text {. }
$$

Then $x^{2} y^{2}=y^{2} x^{2}$ for all $x, y ! \in R$.

Related work appears in [1] and [2].

\section{REFERENCES}

[1] Be11, H.E., On Rings with Commuting Powers, Math. Japonica 24 (1979), 473478 .

[2] Hongan $M$ and Tominaga H., A Commutativity Theorem for S-Unital Rings, Math. J. Qkayama Univ. 21(1979), 11-14.

[3] Nicholson W.K. and Yaqub A., A Commutativity Theorem for Rings and Groups, Canadian Mathematical Bulletin 22(1979), 419-423. 


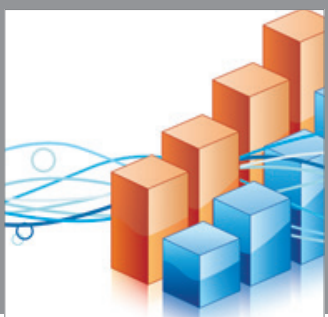

Advances in

Operations Research

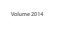

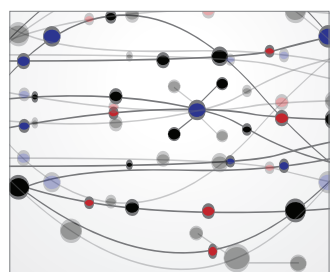

\section{The Scientific} World Journal
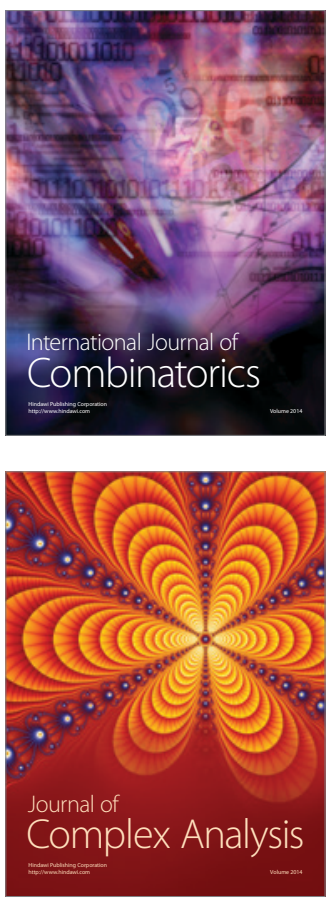

International Journal of

Mathematics and

Mathematical

Sciences
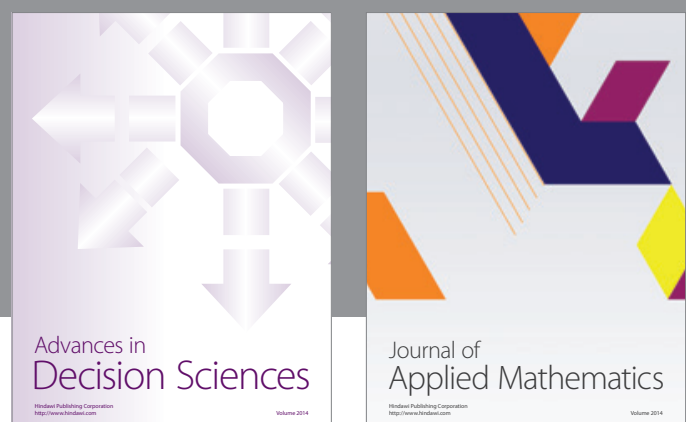

Journal of

Applied Mathematics
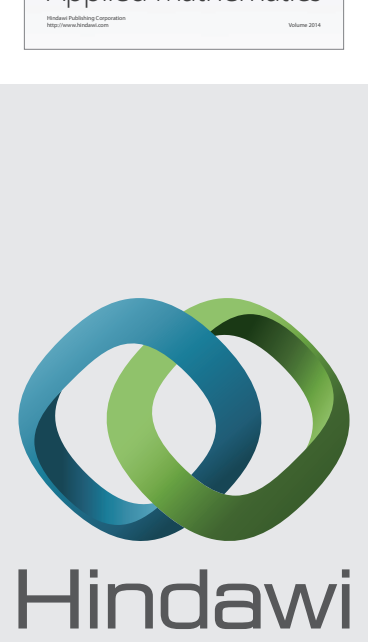

Submit your manuscripts at http://www.hindawi.com
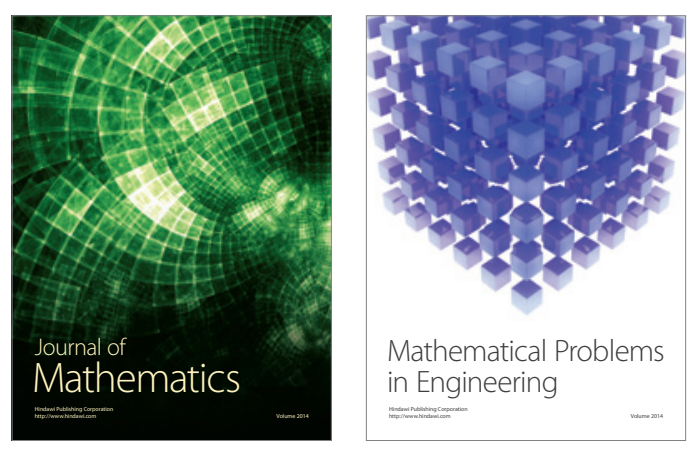

Mathematical Problems in Engineering
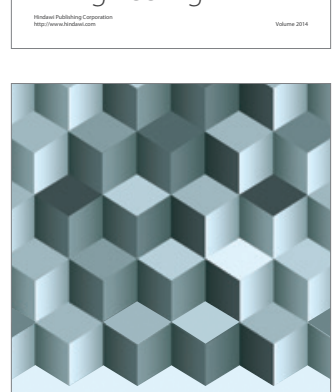

Journal of

Function Spaces
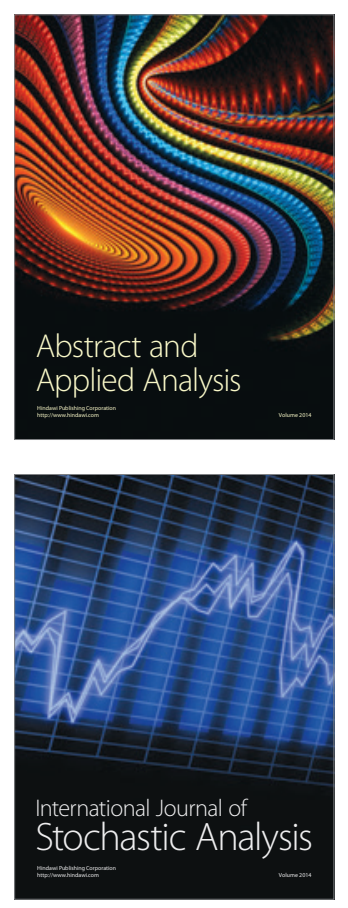

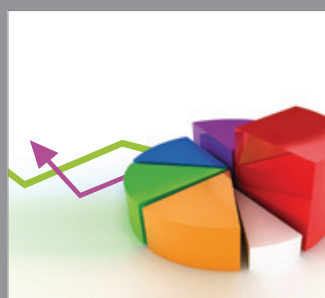

ournal of

Probability and Statistics

Promensencen
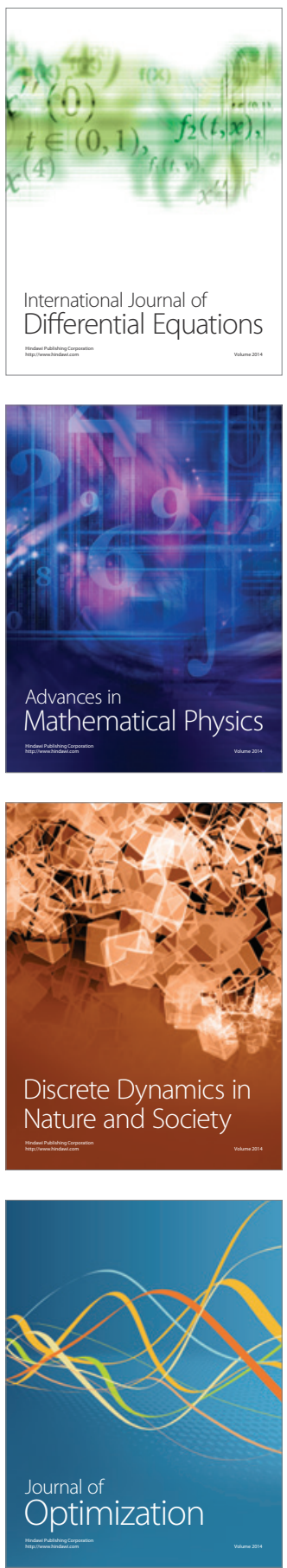